\title{
Extracorporeal Detoxification and Immunocorrection in Treatment of Corona Virus Pneumonia Complications
}

\section{Voinov VA*, Ilkovich MM, Kovalev MG and Voinova YV}

Clinic of Pulmonology, I.P. Pavlov Saint Petersburg First State Medical University, Saint Petersburg, Russia

*Corresponding Author: Voinov VA, Head of the Therapeutic Apheresis Department, Clinic of Pulmonology, I.P. Pavlov Saint Petersburg First State Medical University, Saint Petersburg, Russia.
Received: April 09, 2020

Published: April 28, 2020

(C) All rights are reserved by Voinov VA., et al.

\begin{abstract}
The new corona virus pneumonia caused by COVID-19 having begun in China, has now reached almost all countries of the world, affecting more than 500,000 people, of which more than 25,000 people have died. Unfortunately, specific measures for both preventing and treating this infection, which has pandemic status, are still lacking, which makes this problem very urgent. In severe pneumonia, acute respiratory distress syndrome (ARDS) develops - toxic pulmonary edema with severe and difficult to correct respiratory failure, which requires an extracorporeal membrane oxygenation (ECMO). Since endotoxemia is the basis for ARDS development, extracorporeal detoxification methods should play a leading role in the treatment of this complication. Moreover, with help of hemosorption, not only detoxification is achieved, but also decontamination (delay and removal of pathogens), and with plasma exchange, the body's immune defense system is restored.

Keywords: Viral Bacterial Pneumonia; COVID-19; Acute Respiratory Distress Syndrome; Toxic Pulmonary Edema; Extracorporeal Detoxification and Immunocorrection; Hemosorption; Plasma Exchange
\end{abstract}

\section{Pathogenesis of acute lung damage}

The pathogenetic mechanisms of the corona viral pneumonia are common to all types of acute respiratory distress syndrome, which occurs in other diseases - sepsis, peritonitis, eclampsia of pregnant women and many others. They consist in development of toxic interstitial and then alveolar pulmonary edema due to impaired permeability of the cell membranes on the basis of endotoxemia $[1,2]$.

To verify this, as early as in the $90 \mathrm{~s}$, a series of experimental clinical trials were conducted at our University. Both with endotracheal and intravenous administration of pneumococci in rabbits, a pattern of endotoxemia developed associated with development of toxic pulmonary edema - ARDS [3].

The experiments showed that in development of the lung respiratory parenchyma lesions that occur on the background of acute pneumonia, it is not the spread of the primary pathological process through the airways, but endotoxemia playing the leading role due to the release of both living microbes and inflammation products into the circulation, resulting in impaired permeability of the endothelial cell membranes with access of not only fluid but also protein to the interstitium and then to the alveoli.

It should be considered that the protein penetrating through the porous endothelium promotes formation of hyaline membranes in alveoli, which even after cure determine the picture of chronic re- spiratory failure in such patients [4]. No less severe injuries occur in the renal parenchyma, leading to acute renal failure, up to anuria. And such changes are still left even after the recovery, leading to development of chronic renal failure $[5,6]$.

Thus, in patients with acute pneumonia, a double type of lung lesion develops - the primary one, depending on the spread of the pathogens through the respiratory tract, and the secondary one, resulting from penetration of microbes and inflammatory products from the primary focus into the bloodstream with toxemia development. Moreover, it is not the epithelium of the respiratory tract that threatens the pulmonary parenchyma, but the blood through the vascular endothelium [7].

All types of these toxic substances damage the permeability of the cell membranes, and not only the lungs, but also almost all other internal organs and tissue structures with disorder of their functional state and of multiple organ failure syndrome development.

Moreover, a number of vicious circles appear when toxic pulmonary edema and hypoxemia stimulate hypoxic disturbances in the membrane permeability; damage to the kidneys contributes to additional fluid retention in the body (edema is stimulated) and toxins (toxemia is increasing); damage to the liver with the suppression of its detoxification function also deepens toxemia; toxic myocardiopathy exacerbates microcirculation disorders in the organs; toxic encephalopathy also leads to the brain disorders, and the released 
neuropeptides stimulate neurogenic pulmonary edema. It is this "summation" of lesions in multiple organ failure that determines the extremely high mortality rate - up to $80 \%$ [8]. This syndrome of multiple organ failure reflects a biological catastrophe, a type of biological suicide that occurs in a wide range of clinical situations.

Interstitial and alveolar toxic pulmonary edema blocks the gas exchange at the alveolar level due to the thickening of the airborne barrier (alveoli-capillary membrane). This leads to severe and difficult to correct parenchymal respiratory failure, which is a leading factor in thanatogenesis.

Toxic products circulating in the blood have a damaging effect not only on the endothelium of the blood vessels, but also on the ingredients of the blood itself, mainly on its cells. In particular, activation of platelets contributes to their adhesion and production of their micro-aggregates, which become a kind of foci for the subsequent formation of DIC reactions cascade, stimulating microthrombosis and bleeding.

Thus, ARDS is a secondary toxic lesion of the respiratory parenchyma that occurs in diseases not only of the lungs, but also in a number of other pathological conditions that have common pathogenetic mechanisms. The main one is the toxic disorder of the cell membranes permeability.

\section{ARDS treatment}

Traditional approaches to the treatment are largely determined by the above mentioned diagnoses and resulting in inadequate assessment of this complication causes. When only the inflammatory component of pathogenesis is considered to be the cause, efforts are directed towards providing antibiotic therapy, and the search for new, more powerful antibiotics of "super-wide" action. It is believed that the process progression is associated only with the low sensitivity of pathogens to antibiotics.

Naturally, it would be unreasonable to reject the use of antibiotics in cases where the microbial flora is the main etiological factor. It is also necessary to increase the body's resistance using vitamins, immunostimulating drugs, as well as cardiotonics, membrane stabilizers, antioxidants, and antiplatelet agents.

However, even the most effective antibiotics, killing microbes, are not able to eliminate microbial toxins, and even the microbial bodies themselves require a special elimination system, and in conditions of reduced phagocytic activity, they are retained in the body and continue their harmful effects. The fact of addition and activation of a microbial infection already indicates weakening of the body's defenses, its inability to cope with the pathological condition on its own. Respiratory viral infection contributes even more to immunosuppression, especially in patients weakened by previous chronic diseases, and intoxications.
Since no specific treatment methods for ARDS have been found so far, there is a hope left only for prolonged mechanical ventilation (PMV) [9,10]. Oxygen therapy does not cause any doubts and objections, since the expansion of the alveolo-capillary membrane during edema dramatically slows the diffusion of oxygen through it. However, the hope to restore the gas exchange function of the lungs using PMV seems rather illusory, since it is indeed able to correct ventilation respiratory failure, but diffusion disorders at the alveoli level make it unsuccessful in case of parenchymal respiratory failure. Resuscitators worldwide still hope to adjust some special ventilation conditions, in particular, to increase airway pressure at the end of exhalation (PEEP).

Maintaining such a pressure at the level of $5-10 \mathrm{cmH}_{2} \mathrm{O}$ at some point proves to be effective due to the over-inflation of the alveoli not yet completely filled with effusion, improving the gas exchange [11]. However, special physiological studies show that the volume of the intravascular fluid of the lungs does not only decreases, but even increases due to the greater porosity of the overgrown alveolocapillary membrane, increased filtration area and the affected lymphatic drainage from the pulmonary parenchyma in the result of increasing intrathoracic pressure. It is known that PMV even with ventilation disorders itself stimulates fluid retention in the lungs, inhibits diuresis, and promotes lung barotrauma.

In addition, pneumonia and sepsis, which develop not only in the result of microbial insemination of the respiratory tract, but also in systemic inflammatory response syndrome (septic shock) with release of cytokines such as interleukins 6 and 8 (IL-6, IL-8), as well as tumor necrosis factor $\boldsymbol{\alpha}$ (TNF- $\boldsymbol{\alpha}$ ). It was noted that their level increase occurs 3 - 4 days before of pneumonia developed $[12,13]$. The onset of pneumonia on the background of ARDS is difficult to diagnose, since its signs such as leukocytosis, high fever and radiologically determined changes (lung infiltration) already exist in ARDS with no concomitant infection. On the other hand, endobronchial and pathomorphological studies show the presence of respiratory tract infection on days $2-6$, and signs of pneumonia on days 5 - 12 of ARDS development.

These facts made scientists back in the seventies of the XX century to turn to the use of extracorporeal gas exchange with the help of ECMO. In animal experiments, it was possible and safe to maintain gas exchange for up to three weeks with membrane oxygenators. This gave reason to use them for auxiliary ECMO in acute parenchymal respiratory failure.

The first results of ARDS treatment using ECMO were quite encouraging. Indeed, immediately after connection of membrane oxygenators the gas exchange was restored, and the patients' condition was stabilized. However, there was no inverse significant dynamics of pathological changes in the lungs observed. After the procedure, inflammatory and destructive processes continued to progress. In recent years, ECMO effectiveness has increased up to $47-60 \%$ $[14,15]$. 
Positive results require up to 16 and even 120 days of ECMO $[16,17]$. During this period, it was necessary to transfuse significant amounts of donated plasma, platelet concentrates, to perform parenteral nutrition, round-the-clock monitoring and maintenance by highly qualified specialists, which required considerable financial costs, well exceeding \$100,000. Given such difficulties and ECMO operations complexity, they are not widely used. However, ECMO has taken a definite place among ARDS treatment methods.

Nevertheless, conventional methods of intensive care with various options for mechanical ventilation are still most often used [18].

The presented analysis shows the complexity of ARDS treatment, but at the same time there is almost no pathogenetic approach to its therapy that is ignoring the fact of the endotoxic nature of the lungs and other organs damage and, as a result, detoxification methods are not used.

Our own initial attempts to use ECMO in ARDS did not give the expected results due to the inability to suspend the progression of pathological processes in the lungs and multiple organ failure, despite the correction of the impaired gas exchange during the operation.

These failures, on the one hand, and the results of experimental studies that showed the toxic nature of the lesions of the lungs and other organs, on the other hand, convinced us to consider the use of detoxification methods to be necessary. Under these conditions, only direct blood extracorporeal detoxification methods can stop the process progression and break many formed vicious circles.

\section{Extracorporeal detoxification methods in ARDS}

During hemosorption when up to 3 - 4 circulating blood volume (CBV) passes through a column with carbon sorbents there was quite a complete elimination of many pathological products and even live bacteria were retained and fixed, which was the only truly effective treatment due to the inadequacy of the antibiotic therapy. The level of medium molecular oligopeptides, blood toxicity as a whole (considering the survival time of protozoa) decreased, the general condition improved, the changes in the lungs visualized by the X-ray examination underwent reverse development $[3,7]$.

Nevertheless, in an advanced lung damage only ECMO with 25 - $30 \%$ speed of the minute volume of the blood flow and lasting up to two days made it possible to gain time, i.e. to maintain the gas exchange at a minimum adequate level and during this time to provide more active detoxification. Only such a combination of massive detoxification (up to three hemosorption procedures per day) on the background of ECMO made it possible to ensure the reverse the organ lesions development in extremely severe stages of ARDS. Of the ten such completely hopeless patients they managed to save seven [7].
It should be noted that it usually takes 2 - 3 weeks to treat ARDS with ECMO, while in our cases it took only 15 to 44 hours to stop ARDS of the same extremely severe degree. And the difference in treatment tactics is only in one thing: we have conducted intensive detoxification with hemosorption on the background of ECMO which is still ignored.

There is a review of the treatment of another 44 patients with severe ARDS treated during the period 1998-2015, of whom 15 patients being treated with conventional methods of drug therapy, and 29 patients with comparable severity of ARDS being detoxified by hemosorption or plasmapheresis.

Hemosorption was carried out with 1 - 2 volumes of the circulating blood passing through the columns. Membrane plasmapheresis was performed using Russian PFM-800 or "Rosa" plasma filters on the "Hemofenix" device (Trekpor Technology, Moscow) in the plasma exchange mode with removal of $1.2-2.5 \mathrm{~L}$ or $0.5-1,0$ of the circulating plasma volumes (CPV) and replacing freshly frozen donor plasma. At the same time, laser irradiation of blood was performed.

All the patients received traditional drug therapy, and in case of respiratory failure developing - PMV, in severe cases with PEEP. Moreover, if in the main group of 29 patients 21 were saved $(72.4 \%)$, in the control group of 19 patients only 6 survived (31.6\%).

Here is our clinical observation, which is an example of such a leading role of endotoxemia and immunosuppression in the onset and development of life-threatening ARDS condition and the effectiveness of detoxification.

A total lung lesion with severe multiple organ failure developed in a 40-year-old patient $\mathrm{S}$. with sarcoidosis who had been using hormonal drugs for a long time. Gradually increasing immunosuppression determined the scale and speed of progression of viral respiratory infection. By the beginning of apheresis therapy, the patient was in an extremely serious condition. PMV with PEEP did not correct parenchymal respiratory failure and hypoxic coma An X-ray examination revealed intense and almost total shading ("hepatization") of the lungs. Hepatic-renal failure was manifested by significant fluid retention in the body with an increase in creatinine, bilirubin and transaminases. Central hemodynamics was supported by sympathomimetics, frequent group polytopic ventricular extrasystoles were detected.

At the first stage of extracorporeal detoxification, hemosorption with blood laser irradiation was performed. The very next day, the condition stabilized somewhat. Received $500 \mathrm{ml}$ of urine. An X-ray examination showed signs of airiness in the upper parts of the lungs. The normal rhythm with isolated extrasystoles was practically restored, sympathomimetics were canceled. 
Against this background, a second session of extracorporeal detoxification was performed - membrane plasmapheresis with the exchange of $2000 \mathrm{ml}$ of freshly frozen donor plasma, also with blood laser irradiation. The next day, there was a restoration of consciousness and independent breathing, followed by a more rapid restoration of the functional state of the lungs and other vital organs and a complete recovery.

\section{Discussion}

As the above mentioned findings show in severe ARDS stages the use of extracorporeal detoxification methods affects the overall outcome of the disease. It should be noted that the earlier the detoxification methods are used, the more pronounced is their effectiveness. In this case, as a rule, only one hemosorption or plasmapheresis procedure with blood laser irradiation is sufficient to improve the course of the disease dramatically, so that the body itself can further cope with the complications even having a lower level of drug support. In a severe degree of ARDS, it is often required to repeat another two or three detoxification procedures to achieve stabilization and reverse development of the lung lesions, but if their use is delayed, not all the patients can be saved.

In an extremely severe degree of ARDS with almost total lung damage, severe parenchymal respiratory failure develops that cannot be stopped by any means using PMV. In these cases ECMO provides faster normalization of the gas exchange, and simultaneous intensive detoxification (up to three procedures per day) helps eliminate toxic pulmonary parenchyma edema with restoration of the lungs "airiness" degree during X-ray examination after 7 - 15 hours. And by the time ECMO is completed it is possible to restore a completely satisfactory level of gas exchange function of the lungs [7].

Nevertheless, detoxification alone, achievable by hemosorption, is also not enough for a full therapeutic effect, since the body remains in a state of immunosuppression, due to which there is a possibility of developing this serious complication. A more stable result is achieved by plasma exchange with replacement of the patient's removed plasma with "incompetent" antibodies, immunoglobulins, complement, opsonins with freshly frozen donor plasma. These immune components immediately begin to fight pathogens and other pathological products.

It should be noted that this approach improves not only the humoral, but also cellular immunity, since without complementation there is no opsonization of macrophage receptors, without which the capture and subsequent destruction of pathogens is impossible. This provides a more reliable result, especially when replacing plasma in a volume that is almost equal the patient's CPV.

Massive plasma exchange leads to a more rapid improvement of homeostasis. To compare with hemosorption there is not only a more reliable and complete removal of all the pathological products, regardless of their electrochemical activity, but also a more complete restoration of all plasma components such as proteins with normalized oncotic pressure and volemic balance, hormonalenzymatic activity with restoration of autoregulation mechanisms. All this enables to completely prevent the dramatic scenario of ARDS development and provides a faster and more complete reverse development of toxic pulmonary edema, and other organ disorders, a more complete restoration of their functions and, ultimately, the recovery [19].

Detoxification, which helps to eliminate vascular porosity, is a pathogenetically more justified method of ARDS treatment, since after the cessation of toxic substances penetration into the alveoli, reproduction of the natural surfactant is restored in the coming hours, which eliminates the need for its exogenous preparations administration.

For a more complete restoration of the immune mechanisms, it is also advisable to combine both hemosorption and membrane plasmapheresis with methods of laser blood irradiation (photohemotherapy).

It seems that it is more reasonable to conduct an active detoxification therapy that is hemosorption or/and plasmapheresis. And indeed, almost always after the removal of the "toxic burden" from the kidneys, their excretory function is restored. The very next day diuresis is at least $500-700 \mathrm{ml}$. At the same time, the functional state of other vital organs such as the lungs, liver, heart, and brain is improved [19].

If an acute liver failure added, there is an increase in the level of bilirubin and transaminases such as ALT and AST. And the removal of the "toxic burden" from hepatocytes quickly normalizes the liver function. With an extreme degree of hypoxia, damage occurs to the brain structures up to a deep hypoxic coma. Disorders of both central and peripheral circulation are increasing.

In an extremely severe course of ARDS with addition of multiple organ failure hemodynamics disorders occur, which makes it difficult to carry out any extracorporeal detoxification procedures. However, the existing Russian "Hemofenix" devices, which make it possible to carry out both plasmapheresis with "Rosa" plasma filters and hemosorption with any available hemosorbents even in unstable hemodynamics. This became possible, given the small filling volume of the extracorporeal circuit of this device not exceeding 65-70 ml, which makes it possible to carry out such treatment even in children.

Therefore, the treatment of such patients with ARDS in an extremely serious condition is a rather difficult task. Recently, we have developed treatment tactics taking into account all the aspects of ARDS and multiple organ failure pathogenesis.

Given the high probability of a number of pathogens circulating in the blood, at the first stage it is advisable to resort to massive hemosorption. It provides not only significant detoxification when 1 - $2 \mathrm{CBV}$ is passed through the sorption column, but also decon- 
tamination that is retention of pathogens, both living and dead on the sorbent. This leads to stabilization of the patients' condition when the functions of not only the lungs, but of all other vital organs are improved.

On the second stage, the very next day, you can start plasmapheresis removing at least $60-80 \%$ of the $\mathrm{CPV}$ and replacing with an equal volume of freshly frozen donor plasma. Moreover, in addition to detoxification, there is a correction of the immune defense system with restoration of not only humoral, but also cellular immunity. Very often these hemosorption and plasmapheresis procedures are enough for the body to restore its autoregulation systems and recover from the critical state.

It should only be taken into account that a high risk of bleeding requires special tactics to use anticoagulants. First of all, heparin is completely eliminated and prevention of blood clotting in the extracorporeal circuit is provided by sodium citrate solutions (ACDA).

Nevertheless, one should not wait for such extremely severe conditions to occur and conduct adequate treatment on the early stages of ARDS, when the negative dynamics of its development are already emerging. At the same time, in some cases it is necessary to take into account a rather rapid increase in ARDS severity, when in just a few hours you can "loose" such a patient.

\section{Conclusion}

Thus, both experimental and clinical studies convincingly evidence the toxic nature of ARDS, which makes it reasonable to conduct various methods of extracorporeal detoxification. At the first stage, it is advisable to carry out sorption detoxification, which enables to remove not only toxic metabolites, but also pathogens. Then, during plasma exchange, the removal of toxic products is continued followed by replacement of freshly frozen donor plasma and restoration of the immunity. It is the only way that can help achieve favorable results, especially considering the ineffectiveness of any drug therapy. In addition, it should be emphasized that such measures are necessary to be taken without waiting for the development of severe ARDS, which will provide a more complete recovery of the patients without the risk of residual effects arising in the hyaline membranes syndrome associated with chronic respiratory failure. All the above mentioned is fully useful in cases of ARDS in the new coronavirus infection.

\section{Bibliography}

1. Buttenschoen K., et al. "Endotoxemia and endotoxin tolerance in patients with ARDS". Langenbeck's Archives of Surgery 393.4 (2008): 473-478.

2. Inagawa R., et al. "Ultrastructural Alteration of Pulmonary Capillary Endothelial Glycocalyx During Endotoxemia”. Chest 154.2 (2018): 317-325.
3. Voinov VA., et al. "Issues of the pathogenesis and treatment of respiratory distress syndrome in viral-bacterial pneumonia". In: "Immunology and pathogenesis of influenza and flu-like diseases", Leningrad (1991): 82-87.

4. Ohtsuki Y., et al. "Early Stages of Hyaline Membrane Formation Detected in Alveolar Mouths in Diffuse Alveolar-DamageAssociated Diseases: A Detailed Immunohistochemical Study". International Journal of Surgical Pathology 23.7 (2015): 524530.

5. Basu RK. "Acute kidney injury in hospitalized pediatric patients". Pediatric Annals 47 (2018): e286-e291.

6. Voinov VA., et al. "Therapeutic Apheresis in Acute Kidney Injury". American Journal of Kidney Diseases 4.3 (2018): 1000172.

7. Voinov VA., et al. "Extracorporeal membrane oxygenation and hemosorption in the treatment of severe forms of respiratory distress syndrome]". Efferentnaya Therapia 1.1 (1995): 36-39.

8. Gotloib L. "Hemofiltration in multiorgan failure syndrome secondary to sepsis: a critical analysis of heterogeneitry". Nephron 73.2 (1996): 125-130.

9. Fan E., et al. "Acute Respiratory Distress Syndrome: Advances in Diagnosis and Treatment". The Journal of the American Medical Association 319.7 (2018): 698-710.

10. Patel B., et al. "Extracorporeal membrane oxygenation as rescue therapy for severe hypoxemic respiratory failure". Journal of Thoracic Disease 11.14 (2019): S1688-S1697.

11. Badet M., et al. "Comparison of optimal positive end-expiratory pressure and recruitment maneuvers during lung-protective mechanical ventilation in patients with acute lung injury/ acute respiratory distress syndrome". Respir Care 54.7 (2009): 847-854.

12. Chang I and Schibler A. "Ventilator Associated Pneumonia in Children”. Paediatric Respiratory Reviews 20 (2016): 10-16.

13. Weiterer S., et al. "Sepsis in mechanically ventilated patients with spinal cord injury: a retrospective analysis". Spinal Cord 57.4 (2019): 293-300.

14. Maclaren G and Butt W. "Extracorporeal membrane oxygenation and sepsis". Critical Care and Resuscitation Journal 9 (2007): 76-80.

15. Zabrocki LA., et al. "Extracorporeal membrane oxygenation for pediatric respiratory failure: Survival and predictors of mortality". Critical Care Medicine 39.2 (2011): 364-370.

16. Dadras M., et al. "Extracorporeal membrane oxygenation for acute respiratory distress syndrome in burn patients: a case series and literature up date". Burns Trauma 7 (2019): 28. 
17. Xia J., et al. "Spontaneous breathing in patients with severe acute respiratory distress syndrome receiving prolonged extracorporeal membrane oxygenation". BMC Pulmonary Medicine 19.1 (2019): 237.

18. Bos LD., et al. "ARDS: challenges in patient care and frontiers in research". European Respiratory Society 27 (2018): 147.

19. Voinov VA. "Therapeutic apheresis". Celebris, Constanta (Romania) (2016): 403.

\section{Assets from publication with us}

- Prompt Acknowledgement after receiving the article

- Thorough Double blinded peer review

- Rapid Publication

- Issue of Publication Certificate

- High visibility of your Published work

Website: www.actascientific.com

Submit Article: www.actascientific.com/submission.php

Email us: editor@actascientific.com

Contact us: +919182824667 